\title{
COMPARISON OF ALTERNATIVE IMAGE REPRESENTATIONS IN THE CONTEXT OF SAR CHANGE DETECTION
}

\author{
Andreas Schmitt, Anna Wendleder, Birgit Wessel, Achim Roth \\ German Aerospace Center (DLR) \\ German Remote Sensing Data Center (DFD) \\ D-82234 Weßling
}

\section{INTRODUCTION}

Nowadays, SAR systems are widely used for the survey of vulnerable structures because of their independence of weather and illumination. So, the planning of time series of images becomes quite easy. Additionally, in the case of a disaster event a quick image acquisition is possible. With the new SAR satellite modes an image resolution up to one meter (pixel spacing) can be achieved, which is necessary to examine fine structured areas like city centers. Unfortunately, the interpretation of SAR images is still a difficult task due to their geometrical and radiometrical properties. If we do not concentrate on the examination of SAR signatures but on the detection of changes in the SAR signatures apparent in time series of SAR images, the interpretation is simplified. Geometrical influences can be reduced by using images taken from repeat orbits. Problems with noise are solved by using alternative image representations instead of the pixel space. A change detection algorithm based on the Curvelet transform has already been published [1]. In this contribution we like to show how the chosen image representation affects the change detection results in order to give advice which image representation suits which application. The proposed algorithms including Wavelets, Curvelets and Surfacelets have been implemented in the programming environment Matlab and tested on various TerraSAR-X data.

\section{RELATED WORK}

An overview to principal SAR change detection methods, their advantages as well as their disadvantages can be found in [2]. The approaches mainly differ in the handling of the SAR inherent noise. One idea is to use a special combination of filters to exclude small scale changes but to preserve structures [3]. Another idea starts with the fusion of several SAR images of different incidence angles to a "superresolution" image used as presumably noise free reference image for the comparison [4]. As man-made objects, i.e. geometrical particularities that are not captured by the digital terrain model used for the ortho-rectification of the SAR image, have different SAR signatures 
in the single images, they have to be classified and excluded from the change detection process. It is also conceivable to exploit the coherence (phase information) between two SAR images of the same area to map changes [5]. But this approach implies a relatively short repeat-pass time, especially in higher frequencies like X-band, to avoid additional incoherence caused by natural surfaces. Another possibility is to adopt a hidden and a sliding hidden Markov chain model respectively to select areas with changes in reflectivity even from images with different incidence angles $[6,7]$. Although this method allows to process very large images and does not need additional parameter tuning, except the window size, still a lot of research has to be done to improve the preliminary results according to the authors. Our investigations base on a change detection approach that is composed of an image differentiation in the Curvelet coefficient domain and a subsequent image enhancement step [1]. Only the SAR amplitude data is needed. Co-registered complex SAR images in slant range can be assimilated as well as geo-referenced products. The acquisition geometry is not necessarily restricted to repeat orbit acquisitions. So, the crucial point lies in the choice of the alternative image representation. The Curvelet transform used in this approach has originally been developed to describe an object with edges with a minimal number of coefficients in the continuous space [8]. Much research work was done to transfer the definitions from the continuous to the discrete space so that digital image processing becomes feasible [9]. Many applications in different scientific fields have been published so far, e.g. in geo- and astrophysics, that are summarized in [10]. For comparison the Surfacelet transform, that originates from 3D-data processing mainly in a medical context [11], and the meanwhile well-known Wavelet transform using the Daubechies Wavelet [12] have been added.

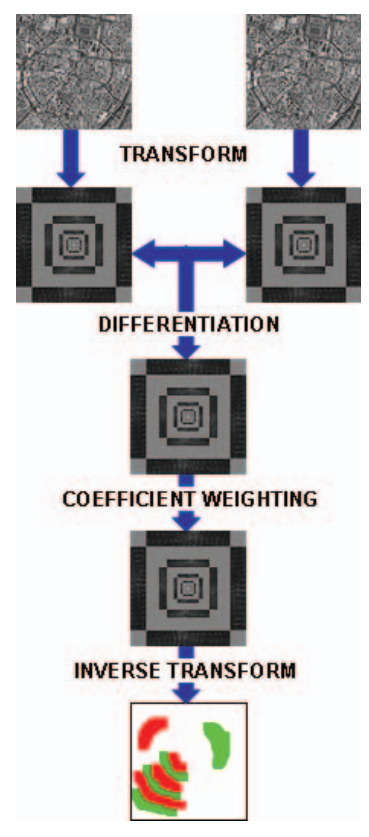

Fig. 1. Flowchart

\section{CHANGE DETECTION APPROACH}

As input two or more co-registered and equally sized SAR images are required. In the case of TerraSAR-X data the high precision geo-location of the delivered geo-coded products EEC and GEC, processed with "science" orbits is sufficient for the coregistration. The algorithm, whose flowchart is depicted in Fig. 1, starts with the transform of the single images from the spatial to the coefficient domain. To analyse the influence of different alternative image representations the 2-dimensional Wavelet transform (Daubechies wavelet), the Curvelet transform and the Surfacelet transform are inserted. Then, the coefficients of an input image pair are differentiated. The procedure continues with an image enhancement step, which weights the intensity of the coefficients in order to suppress weak coefficients, that presumably only represent noise, and to amplify stronger coefficients, that contain the structures of the image. The weighting function is automatically adapted to the image content by an iterative process. 
This image enhancement procedure is suitable for all three image representations. Finally, the difference image is transformed back to the spatial domain, where the positive and negative changes respectively are color-coded red and green to visualize the sign of the detected changes. To get a reference image, this procedure is carried out once for the pixel-based change detection without any transformation so that the image enhancement is done directly on the pixel gray values.

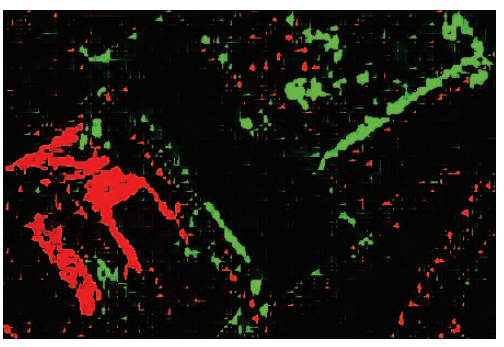

(a) Pixel-wise

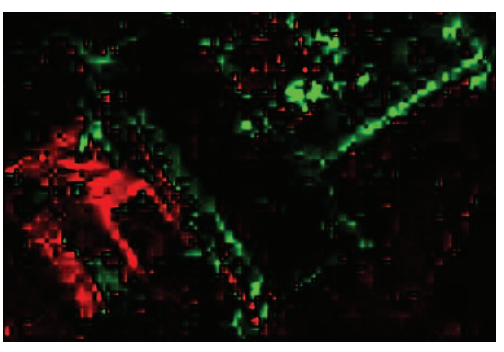

(b) Wavelets pixel-based procedure detects many small "pixel-sized" changes (Fig. 2(a)), as expected. These changes are mostly caused by noise and do not report real changes in the imaged area. If the Wavelets transform is used (Fig. 2(b)), the irregular single pixel changes agglomerate to more regular structures. As the originally 1D-Wavelet transform is applied bi-orthogonally, i.e. once in each direction for each row and column, these structures are oriented only in horizontal and vertical direction. In contrast to that, the Curvelet transform adapts its basic (linear) elements to multiple orientations, so that structures of an arbitrary orientation are well approximated. This effect becomes obvious in the green line in the upper right of the image. While this line is interrupted several times both in the pixel- and Wavelet-based results, it appears as strong smooth line using Curvelets (Fig. 2(c)). Here the linear form of the basic elements becomes clearly visible. The Surfacelet transform (Fig. 2(d)), based on elliptical elements, sums up the single pixel changes to small, smooth and often ellipticalshaped regions. The green line again is interrupted, but the area-like changes, mapped in green in the upper middle, are drawn precisely. One can perceive

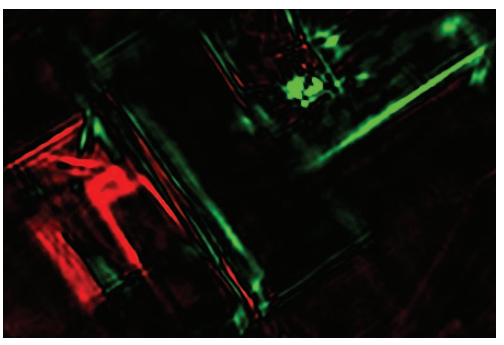

(c) Curvelets

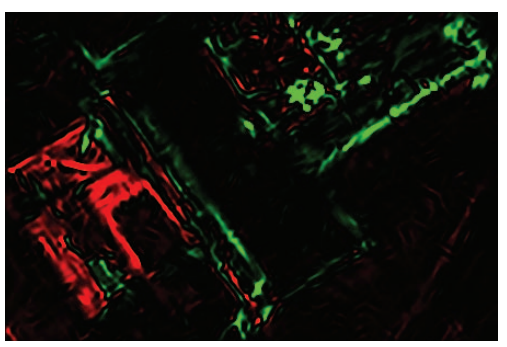

(d) Surfacelets

Fig. 2. Detected changes 01.07.2009-23.07.2009

\section{$R$ : darkened, G: brightened up} that similar to the Wavelet-based results (cf. Fig. 2(b)), the changes mapped in red in the lower right are more fine structured. While the Wavelet transform only holds horizontal and vertical structures, the Surfacelet transform also depicts diagonal and even curved structures. From the comparison of all four images, one can infer that the Curvelet and the Surfacelet transform help to get the irregular changes more smoothly shaped. 


\section{CONCLUSION}

Alternative image representations are very helpful for change detection in SAR imagery. The main goal is the decomposition in different scales, which helps to join several single pixel changes to larger areas without loosing structures. While the results using the Wavelet transform are not much better than the results of a simple pixel-based approach, the Curvelet and the Surfacelet transform approximate the underlying structures very well. Anyhow the geometric form of the basic element is traced in the resulting images in both cases, so that we advise to select the transform according to the geometric form of the changes one is interested in. To detect single cars (e.g.) choose the ellipse-like Surfacelet transform; to detect changes in built-up areas, e.g. new or destroyed buildings, prefer the Curvelet transform, which fits best the linear reflection of the walls. The data preparation, the image enhancement, and the data output can be adopted without major changes.

\section{REFERENCES}

[1] A. Schmitt, B. Wessel, and A. Roth, "Curvelet-based change Detection for man-made Objects from SAR Images," in Proceedings of IGARSS, Kapstadt (South Africa), July 2009.

[2] L. Polidori, S. Caillault, and J.-L. Canaud, "Change detection in radar images: methods and operational constraints," in Geoscience and Remote Sensing Symposium. IGARSS '95. 'Quantitative Remote Sensing for Science and Applications', Florence (Italy), 1995, vol. 2, pp. 1529-1531.

[3] R.G. White, "Change detection in sar imagery," International Journal of Remote Sensing, vol. 12, no. 2, pp. 339-360, 1991.

[4] J. Marcos, R. Romero, D. Carrasco, V. Moreno, J. Valero, and M. Lafitte, "Implementation of New SAR Change Detection Methods: Superresolution SAR Change Detector," Journal for Photogrammetry and Remote Sensing, vol. 57, pp. 327-345, 2003.

[5] P. Wright, T. Macklin, C. Willis, and T. Rye, "Coherent Change Detection with SAR," in European Radar Conference, EURAD, Paris (France), 2005, pp. 17-20.

[6] S. Derrode, G. Mercier, and W. Pieczynski, "Unsupervised Change Detection in SAR Images Using a Multicomponent HMC model," in Second International Workshop on the Analysis of Multitemporal Remote Sensing Images, P.C. Smits and L. Bruzzone, Eds. 2003, pp. 16-18, European Commission Joint Research Centre, Ispra (Italy).

[7] Z. Bouyahia, L. Benyoussef, and S. Derrode, "Change detection in synthetic aperture radar images with a sliding hidden Markov chain model," Journal of Applied Remote Sensing (JARS), SPIE, vol. 2, no. 1, 2008.

[8] E. J. Candès and D. L. Donoho, Curve and Surface Fitting, chapter Curvelets - a surprisingly effective nonadaptive representation for objects with edges, pp. 105-120, Innovations in Applied Mathematics. Vanderbilt University Press, Nashville (TN), Saint-Malo (France), 1999.

[9] E. J. Candès, L. Demanet, D. L. Donoho, and L. Ying, "Fast Discrete Curvelet Transforms," Multiscale Model. Simul., vol. 5, pp. 861-899, 2005.

[10] L. Demanet, “curvelet.org,” http://www.curvelet.org, 2007, (accessed on 10 December 2009).

[11] Y. Lu and M. N. Do, "Multidimensional Directional Filter Banks and Surfacelets," IEEE Transactions on Image Processing, vol. 16, pp. 918-931, 2007.

[12] A. Graps, “An Introduction to Wavelets," IEEE Computer Science Engineering, vol. 2, no. 2, pp. 50-61, 1995. 